\title{
Hypervascular hepatic masses in a patient who underwent the Fontan procedure
}

Min Kyu Kang ${ }^{1}$, Sung Bum Kim ${ }^{1}$, and Joon Hyuk Choi ${ }^{2}$

Departments of ${ }^{1}$ Internal Medicine and ${ }^{2}$ Pathology, Yeungnam University College of Medicine, Daegu, Korea

Received : May 19, 2020

Revised : June 27, 2020

Accepted : September 25, 2020

\section{Correspondence to}

Min Kyu Kang, M.D.

Tel: +82-53-620-3830

Fax: +82-53-623-8038

E-mail: kmggood111@naver.com https://orcid.org/0000-0002-

1435-3312
A 36-year-old woman was referred to our clinic with a 7-day history of epigastric discomfort. According to her treatment history, at the age of 25 years, she had undergone the Fontan procedure for severe pulmonary stenosis and double-outlet right ventricle. She had visited another hospital 3 years before with the same symptoms, and abdominal computed tomography (CT) was performed (Fig. 1A). She had no history of alcohol consumption. Although her physical examination results were unremarkable, the laboratory test results were as follows: alanine aminotransferase, $50 \mathrm{IU} / \mathrm{L}$; alkaline phosphatase, 336 IU/L; gamma-glutamyl transferase, $204 \mathrm{IU} / \mathrm{L}$; albumin, $3.43 \mathrm{~g} /$ $\mathrm{dL}$; and international normalized ratio, 1.3. Laboratory tests for viral hepatitis were negative. Abdominal $\mathrm{CT}$ revealed variable-sized homogenous hypervascular masses in both hepatic lobes without significant abdominal lymphadenopathy (Fig. 1B). Compared with the previous abdominal $\mathrm{CT}$, the number and size of the masses had increased. Upper endoscopy and colonoscopy performed to evaluate the possibility of metastatic cancer revealed normal results.

Histologic examination of the biopsied
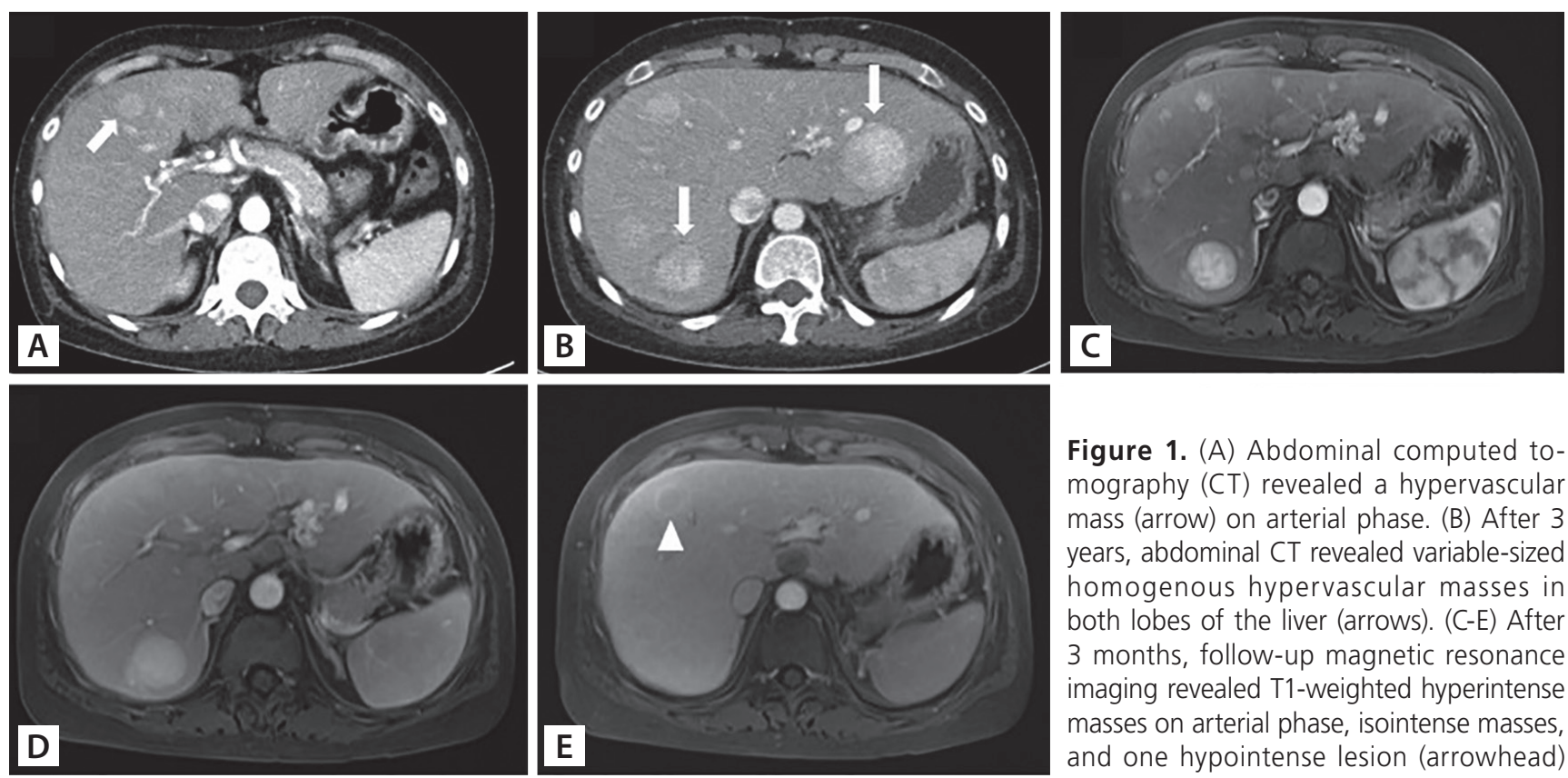

Figure 1. (A) Abdominal computed tomography (CT) revealed a hypervascular mass (arrow) on arterial phase. (B) After 3 years, abdominal $C T$ revealed variable-sized homogenous hypervascular masses in both lobes of the liver (arrows). (C-E) After 3 months, follow-up magnetic resonance imaging revealed T1-weighted hyperintense masses on arterial phase, isointense masses, and one hypointense lesion (arrowhead) on delayed phase, suggested possibility of hepatocellular carcinoma with atypical focal nodular hyperplasia. 

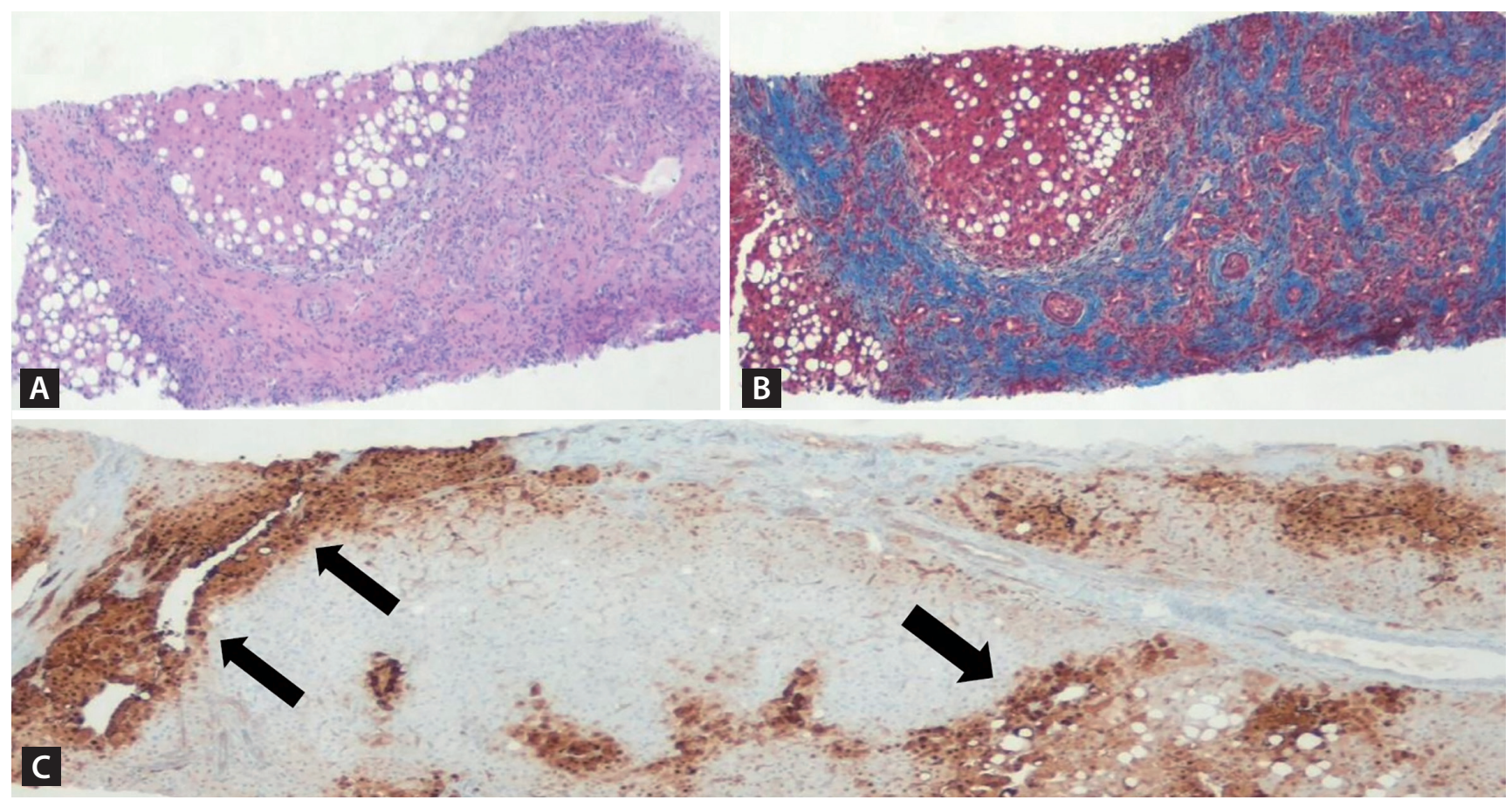

Figure 2. (A, B) Nodular architecture composed of hepatocytes with abundant fibrous septa (A: H\&E, $x 40$; B: trichrome staining, $\times 40$, respectively). (C) Map-like staining pattern (arrows), consistent with focal nodular hyperplasia (glutamine synthetase staining, $\times 40)$.

masses (Fig. 1B, arrows) revealed a nodular architecture composed of hepatocytes with fibrous septa and a map-like staining pattern of glutamine synthetase (Fig. 2C, arrows), consistent with focal nodular hyperplasia (FNH) (Fig. 2). After 3 months, follow-up T1-weighted magnetic resonance imaging (MRI) revealed hyperintense masses on the arterial phase, isointense masses, and a single hypointense lesion (Fig. 1E, arrowhead) in the delayed phase, suggesting the possibility of hepatocellular carcinoma (HCC) with atypical findings of FNH (Fig. 1C-E). Serum alpha fetoprotein was normal at $3.08 \mathrm{ng} / \mathrm{mL}$. However, prompt rebiopsy of the hypointense lesions (Fig. 1E, arrow) on MRI was performed.

The Fontan procedure is a palliative operation in patients with congenital heart disease, including a single ventricle, which directs the inferior vena cava into the pulmonary artery circulation. Hemodynamic alterations in elevated central venous pressure and prolonged congestive hepatopathy are the main causes of Fontan-associated liver disease, including FNH, liver cirrhosis, and HCC. MRI findings of typical FNH are characterized by gradual hyperintensity without washout from the arterial to the delayed phase, whereas those of Fontan-associated FNH could show washout in the delayed phase due to hemodynamic alterations, including congestive hepatopathy. However, in cases with atypical radiological features, biopsy is recommended to exclude the possibility of HCC. In addition, surveillance for liver cirrhosis and HCC in patients who underwent the Fontan procedure is required.

Informed consent was obtained from the patient. This study was approved by the Institutional Review Board of Yeungnam University Hospital (2020-03-035).

\section{Conflict of interest}

No potential conflict of interest relevant to this article was reported.

\section{Acknowledgments}

This research was supported by the Bio \& Medical Technology Development Program of the National Research Foundation (NRF) and funded by the Korean government (2019M3E5D1A02068089). 